\title{
Injectable microparticle-gel system for prolonged and localized lidocaine release. I. In vitro characterization
}

\author{
Pen-Chung Chen, ${ }^{1}$ Yoon Jeong Park, ${ }^{6}$ Li-Chien Chang, ${ }^{2}$ Daniel S. Kohane, ${ }^{3,4}$ Robert H. Bartlett, ${ }^{5}$ \\ Robert Langer, ${ }^{4}$ Victor C. Yang ${ }^{1}$ \\ ${ }^{1}$ Albert B. Prescott Professor of Pharmaceutical Science, College of Pharmacy, The University of Michigan, 428 Church \\ Street, Ann Arbor, Michigan 48109-1065 \\ ${ }^{2}$ School of Pharmacy, National Defense Medical Center, 161 MinChun E. Road, Sec. 6, Taipei, Taiwan \\ ${ }^{3}$ Department of Pediatrics, Massachusetts General Hospital and Harvard Medical School, 55 Fruit Street, Boston, \\ Massachusetts 02114 \\ ${ }^{4}$ Department of Chemical Engineering, 16-343 Massachusetts Institute of Technology, Cambridge, Massachusetts 02139 \\ ${ }^{5}$ School of Medicine, The University of Michigan, Ann Arbor, Michigan 48109-1065 \\ ${ }^{6}$ College of Dentistry, Seoul National University, 28-2 Yongon-Dong, Chongno-ku, Seoul 110-749, Korea
}

Received 26 January 2004; revised 24 March 2004; accepted 2 April 2004

Published online 25 May 2004 in Wiley InterScience (www.interscience.wiley.com). DOI: 10.1002/jbm.a.30086

\begin{abstract}
Current treatment protocol for postoperative pain is to infuse anesthetic solution around nerves or into the epidural space. This clinical practice is beset by the short duration of the anesthetic effect unless the infusion is continuous. Continuous infusion, however, requires hospitalization of the patients, thereby increasing medical costs. In addition, it also causes systemic accumulation of the drug. We reported herein a novel treatment for the postoperative pain by applying to the surgical site a biodegradable microsphere-gel system for prolonged and localized release of encapsulated anesthetic drugs. This lidocaine-containing biodegradable poly(D,L-lactic acid) (PLA) microsphere system, although being established previously by other investigators, was hindered by a burst release and a followed rapid release of the drug within several hours in vitro. In this article, we demonstrated that by a step-by-step modification of the formulation, prolonged release of lidocaine, up to several days in vitro, could be achieved. Differential scan-
\end{abstract}

ning calorimetry revealed a lower glass transition temperature for these lidocaine-loaded microspheres comparing to that of lidocaine-free microspheres. This decreased $T_{g}$ explained for the tendency of the lidocaine-loaded microspheres to physically fuse at higher temperatures. In vitro studies showed that microspheres, when loaded with 35\% lidocaine, yielded a threefold increase in the degradation rate. The molecular weight of PLA of the drug-loaded microspheres was reduced by $50 \%$ within a period of 1 month. Based on the results (of prolonged lidocaine release and rapid PLA microsphere degradation), this lidocaine-loaded PLA microsphere system could offer a simple solution to the treatment of postoperative pain. (c) 2004 Wiley Periodicals, Inc. J Biomed Mater Res 70A: 412-419, 2004

Key words: lidocaine; poly(D,L-lactic acid); biodegradable microspheres; poloxamer; postoperative pains

\section{INTRODUCTION}

Techniques have already been developed to localize anesthetic drugs in the nerve surroundings for the treatment of postoperative pain. Severe chest pain caused by heart surgery can be effectively relieved by the technique of intercostal nerve block. ${ }^{1}$ In performing this technique, an anesthetic, such as lidocaine, is injected locally into the intercostal space of the individual. Although the beneficial effects of this tech-

Correspondence to: V.C. Yang; e-mail: vcyang@umich.edu Contract grant sponsor: NIH; contract grant number: HL 55461

(c) 2004 Wiley Periodicals, Inc. nique have been well documented, ${ }^{2}$ this practice is nevertheless limited by the short duration of the local anesthetic effect that generally lasts for only a few hours.

To achieve long-lasting effects, O'Kelly and Garry introduced the technique of continuous intercostal nerve block by repeated injection of anesthetics through an indwelling catheter located in the intercostal space. ${ }^{3}$ Despite improvement in relieving chest and abdominal pain, this technique nevertheless remains unpopular due to several possible complications; such as accidental systemic absorption of the anesthetic drug caused by misplacement or migration of the catheter, as well as cumulative systemic absorption of the drug after prolonged infusions. 
Epidural infusion of anesthetics has also been established, and remains in major clinical practices. ${ }^{4-6}$ Even with a high safety record, however, cardiovascular effects like hypotension ${ }^{7,8}$ and tachyphylaxis ${ }^{9-12}$ were still reported. Pharmacokinetics data, shown by Sjogren and Wright, ${ }^{13}$ also indicated that the incidence of tachyphylaxis and systemic toxicity resulting from continuous lumbar epidural infusions of lidocaine were significant.

To achieve a more effective and safe treatment for pain, biodegradable microspheres have been applied as a delivery system for prolonged release of the anesthetic drugs. The localized and sustained release of anesthetics provided by such a system would reduce the required drug dose for effective treatment, thereby preventing the possibility of introducing accidental or cumulative systemic drug absorption. Indeed, several investigators ${ }^{14,15}$ have reported the preparation of lidocaine-releasing PLA-based microspheres or nanoparticles. Such preparations, however, have all had a common shortcoming of a large burst release followed by a rapid release of lidocaine within several hours; features that are not suitable for achieving the longterm pain relief.

Herein, we report that by a stepwise modification of the formulation of the PLA microspheres, sustained release of lidocaine for several days was achieved in vitro. Aside from the sustained release feature, these microspheres, prepared from low molecular-weight PLA, also possessed the proper degradation kinetics, thereby avoiding the need for surgical removal. Based on the prolonged lidocaine release and desirable degradation rate, these microspheres could offer an attractive clinical approach in treating postoperative pain.

\section{MATERIALS AND METHODS}

\section{Materials}

Poloxamer 188 (PO188), lidocaine hydrochloride, lidocaine (Lid), and sorbitan monooleate $\left(\mathrm{Span} 80^{\circledR}\right)$ were purchased from Sigma (St. Louis, MO). Poly(D,L-lactic acid) (PLA, iv $\sim 0.4, \mathrm{MW} \sim 20,000$ Daltons) and Poly(vinyl alcohol) (MW $\sim 6000$ Daltons and $80 \mathrm{~mol} \%$ hydrolyzed) were obtained from Polyscience Inc. (Warrington, PA). Tetrahydrofuran (THF, HPLC grade) was purchased from Fisher Scientific (Pittsburgh, PA). Water was distilled and deionized $\left(\mathrm{ddH}_{2} \mathrm{O}\right)$.

\section{Preparation of lidocaine-loaded PLA microspheres}

Microspheres were prepared using the oil/water solventevaporation method. ${ }^{16}$ To investigate the effect of lidocaine loading on the release kinetics, $250 \mathrm{mg}$ PLA and various amounts of lidocaine (75 to $375 \mathrm{mg}$ ) were dissolved in 2.5 $\mathrm{mL}$ methylene chloride. This prepared organic phase was then mixed with $5 \mathrm{~mL}$ aqueous solution containing $10 \%$ $(\mathrm{w} / \mathrm{v}) \mathrm{PO} 188$ and $7 \% \mathrm{NaCl}$, and the mixture was sonicated for 2 min to produce an oil/water emulsion. The emulsion was added to $25 \mathrm{~mL}$ aqueous solution containing 10\% PO188 and $7 \% \mathrm{NaCl}$, stirred at $1700 \mathrm{rpm}$ for $30 \mathrm{~min}$, and followed by evaporation of the solvent (methylene chloride) for 20 min on a rotary evaporator. The microspheres were collected by centrifugation at $4^{\circ} \mathrm{C}$ and then freeze dried.

To examine the effect of the excipient (Span80) on lidocaine release kinetics, various amounts of Span80 (0 to 37.5 $\mathrm{mg}$ ), $250 \mathrm{mg}$ PLA, and $375 \mathrm{mg}$ lidocaine (Lid/PLA = 150\%) were dissolved in $2.5 \mathrm{~mL}$ methylene chloride. Methylene chloride was removed by rotary evaporation with slowly heating the emulsion to $45^{\circ} \mathrm{C}$. All the other experimental conditions remained identical to those described earlier in the preparation of microspheres.

To examine the effect of PLA concentration on lidocaine release kinetics, two formulations of lidocaine-loaded microspheres were prepared. One formulation was identical to that described above in the study of the effect of Span 80 (37.5 mg), whereas the other formulation consisted of twice the amount of all involved constituents (while maintaining the same Lid/PLA and Span80/PLA ratios). The stirring time was extended to $2 \mathrm{~h}$ to naturally evaporate methylene chloride without the use of an evaporator. The microspheres were collected by centrifugation at $4^{\circ} \mathrm{C}$ and then washed twice with $25 \mathrm{~mL}$ of $\mathrm{ddH}_{2} \mathrm{O}$. All the other experimental conditions remained identical to those described earlier in the preparation of microspheres.

Lidocaine-free microspheres were prepared similarly to those made above (500 $\mathrm{mg}$ PLA and $75 \mathrm{mg}$ Span80) except in the absence of lidocaine. All the microsphere preparations were stored at $4^{\circ} \mathrm{C}$ under vacuum immediately following their fabrication.

\section{Characterization of microspheres}

\section{Surface morphology and particle size}

The morphology and average particle size of the prepared microspheres were examined by using a Philip FEG scanning electron microscope (SEM). The average size was estimated from the diameter of 100 randomly selected microspheres.

Assay for lidocaine content

An Alltech (Deerfield, IL) reverse-phase high-pressure liquid chromatography system (RP-HPLC) equipped with a Prosphere C-18 RP-column was used to measure lidocaine concentrations. Twenty milligrams of microspheres was dissolved in $5.5 \mathrm{~mL}$ of acetonitrile, followed by the addition of $9.5 \mathrm{~mL}$ of $0.05 \mathrm{M} \mathrm{NaH}_{2} \mathrm{PO}_{4}$ solution to precipitate the PLA polymer. After filtration, $100 \mu \mathrm{L}$ of the filtrate was applied to a reverse-phase HPLC equipped with a $C_{18}$ column at a flow rate of $1 \mathrm{~mL} / \mathrm{min}$. The column was eluted using $0.05 \mathrm{M}$ 
$\mathrm{NaH}_{2} \mathrm{PO}_{4}$ solution containing $36.5 \%$ acetonitrile. The elution peak was identified by using a UV detector at $215 \mathrm{~nm}$.

The amount of injected lidocaine was estimated from a preconstructed calibration curve. The loading content of lidocaine in the microspheres was calculated as follows:

Lidocaine loading content (\%)

$$
=\frac{\text { weight of lidocaine in the microspheres }}{\text { total weight of the microspheres }} \times 100 \%
$$

The efficiency of lidocaine encapsulation was defined as the weight percentage of the loaded lidocaine relative to the initial dissolved lidocaine in the organic phase.

Glass transition temperature of microspheres

Measurements of the phase transition temperature of lidocaine, PLA, and microspheres of the various lidocaine loadings were carried out using a PerkinElmer (Wellesley, MA) Model 7 Differential Scanning Calorimeter (DSC) at a scanning rate of $10^{\circ} \mathrm{C} / \mathrm{min}$ from 20 to $100^{\circ} \mathrm{C}$. Melting points $\left(T_{m}\right)$ and glass transition temperatures $\left(T_{g}\right)$ were determined as the temperature at the onset of the peaks.

\section{In vitro lidocaine release from the microspheres}

A double-layer folded sieve box containing $50 \mathrm{mg}$ of the microspheres was immersed in a closed vial containing $5 \mathrm{~mL}$ of $0.1 \mathrm{M}$ phosphate buffer ( $\mathrm{pH} 7.4$ ). The vial was then placed in a shaken water bath regulated at $30 \mathrm{rpm}$ and $37^{\circ} \mathrm{C}$. At various times, the entire buffer was collected and replaced with the same volume of a fresh buffer. Aliquots containing $100 \mu \mathrm{L}$ of the collected buffer were then applied to RP-HPLC and assayed for the amount of released lidocaine. At the end of the experiment, the microspheres were collected, freeze dried, and assayed for the remaining lidocaine. Mass balance was conducted to verify that the amount of released and remaining lidocaine matched that of the initial loading dose.

\section{Degradation studies of the PLA microspheres}

\section{PLA molecular weight changes}

The molecular weights of PLA in both lidocaine-free and -loaded microspheres after incubation were determined by gel permeation chromatography using a Styragel column (Waters, Milford, MA). In brief, $15 \mathrm{mg}$ of the microspheres was placed into a closed vial containing $5 \mathrm{~mL}$ of $0.1 \mathrm{M}$ phosphate buffer ( $\mathrm{pH}$ 7.4) and incubated in a shaken water bath at $37^{\circ} \mathrm{C}$. At various times, the microspheres were collected by centrifugation and washed twice with $5 \mathrm{~mL}$ of $\mathrm{ddH}_{2} \mathrm{O}$. The microspheres were freeze dried, dissolved in THF, and then assayed for their molecular weights. The peak, weight-averaged, and number-averaged molecular weights $\left(M_{p}, M_{w}\right.$, and $M_{n}$, respectively) of PLA were calcu- lated using the PL Caliber ${ }^{\oplus}$ Software (Polymer Laboratories, Amherst, MA).

\section{Surface morphology changes}

Surface morphology of the PLA microspheres after incubated at $37^{\circ} \mathrm{C}$ for various time periods was examined by SEM according to the procedures described earlier.

\section{RESULTS AND DISCUSSIONS}

\section{Characterization of microspheres}

\author{
Morphology of microspheres
}

The lidocaine-free microspheres showed a smooth surface [Fig. 1(a)], whereas lidocaine-loaded microspheres exhibited a porous surface [Fig. 1(b)], with the size of both types of microspheres being seen mostly in the range of $1-50 \mu \mathrm{m}$. Inside the microspheres, however, both the lidocaine-free and -loaded microspheres displayed porous structures, as observed by SEM (data not shown). Formation of such porous structures could be accounted for by the occurrence of a phase separation in the PLA-dissolving organic phase that was driven by a solvent (methylene chloride)-nonsolvent (water) exchange process; a phenomenon discussed previously by Witte and coworkers. ${ }^{17}$

Effects of the preparation method on the loading content, encapsulation efficiency, and size of the microspheres

Table I summarizes the results of the loading content and encapsulation efficiency of all the prepared microspheres. As shown by the results in Experiments 1 to 3, the encapsulation efficiency increased with increasing the initial lidocaine concentration in the organic phase. Under a microscope, it was observed that the higher the initial lidocaine concentration, the more lidocaine crystals were formed in the water phase. This observation implied that the water phase was saturated by lidocaine that had leached out from the microspheres. As a consequence, diffusion of lidocaine out of the microspheres was retarded during the fabrication process, resulting in higher encapsulation efficiency for microspheres prepared with higher initial lidocaine concentrations (e.g., Experiment 3). Because the increase in temperature could significantly increase the solubility and diffusion of lidocaine in water, the encapsulation efficiency decreased when the microsphere emulsions were heated to evaporate 

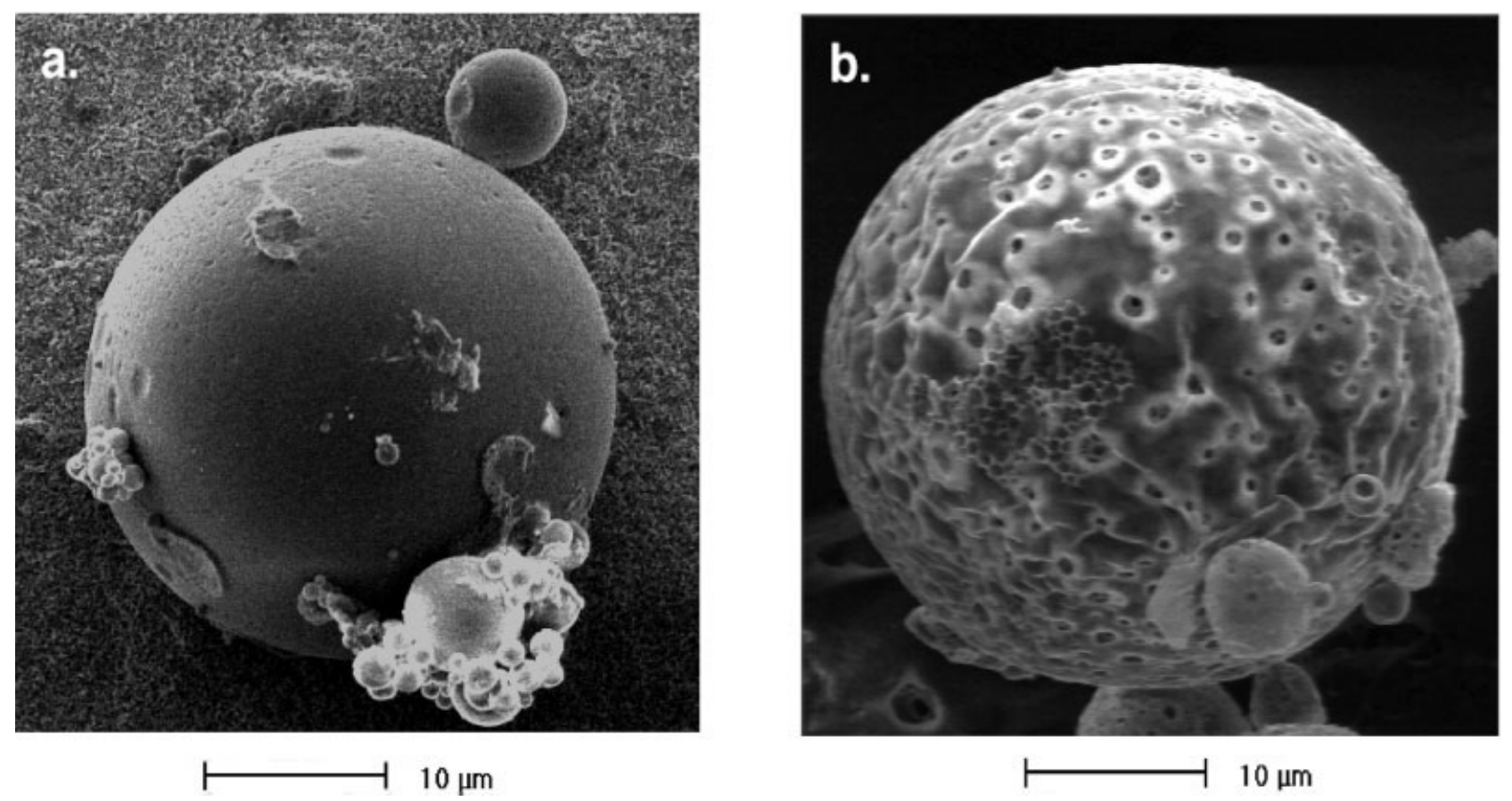

Figure 1. The SEM photographs of: (a) lidocaine-free microspheres; and (b) lidocaine-loaded microspheres. Samples were selected from Experiment 8 in Table I.

the methylene chloride solvent (e.g., compare Experiment 3 with Experiment 4). The use of Span80, however, did not exert any obvious effects on either the loading content or the encapsulation efficiency (see Experiments 4 to 6). On the other hand, the extended stirring employed in Experiment 7 had allowed more lidocaine crystals to dissolve and subsequently diffuse out of the microspheres, and consequently, resulted in a reduction of encapsulation efficiency when the results were compared with those seen in Experiment 6. In addition, results in Experiments 7 and 8 showed that the loading content was increased by about threefold when the concentration of both PLA and lidocaine was doubled. Nevertheless, altering the preparation method did not seem to change the average size of the prepared microspheres (ranging from 7 to 9 $\mu \mathrm{m})$, except in Experiment 8 when a significant increase in the microsphere size (i.e., to $14 \mu \mathrm{m}$ ) was observed with doubled PLA concentration. This increase in size could be due to an increase in the viscosity of the organic phase at the higher PLA concentration.

TABLE I

Effects of Preparation Methods on the Loading Content and Encapsulation Efficiency of the Microspheres

\begin{tabular}{|c|c|c|c|}
\hline Release Expt. Set & Expt. Number & Loading Content $(\%)^{\mathrm{a}}$ & Encapsulation Efficiency $(\%)^{\mathrm{b}}$ \\
\hline \multirow[t]{3}{*}{ Varying Lid/PLA } & $1^{\mathrm{c}}(\mathrm{Lid} / \mathrm{PLA}=30 \%)$ & $3.8 \pm 0.5$ & $13.1 \pm 1.6$ \\
\hline & $2^{c}(\mathrm{Lid} / \mathrm{PLA}=100 \%)$ & $16.2 \pm 0.8$ & $19.4 \pm 1.1$ \\
\hline & $3^{c}(\mathrm{Lid} / \mathrm{PLA}=150 \%)$ & $28.5 \pm 1.7$ & $27.3 \pm 0.8$ \\
\hline \multirow[t]{3}{*}{ Varying Span80/PLA } & $4^{\mathrm{d}}($ Span $80 /$ PLA $=0 \%)$ & $17.9 \pm 0.6$ & $14.5 \pm 0.6$ \\
\hline & $5^{\mathrm{d}}($ Span $80 / \mathrm{PLA}=5 \%)$ & $18.1 \pm 1.2$ & $14.8 \pm 1.2$ \\
\hline & $6^{\mathrm{d}}($ Span $80 /$ PLA $=15 \%)$ & $19.8 \pm 0.3$ & $16.5 \pm 0.3$ \\
\hline $\begin{array}{l}\text { Doubling PLA, Lid, } \\
\text { and Span } 80\end{array}$ & $7^{\mathrm{e}}(\mathrm{PLA}=10 \%, \mathrm{Lid}=15 \%, \operatorname{Span} 80=1.5 \%)$ & $10.5 \pm 1.0$ & $7.8 \pm 0.8$ \\
\hline $\begin{array}{l}\text { concentrations in } \\
\text { the organic phase }\end{array}$ & $8^{\mathrm{f}}(\mathrm{PLA}=20 \%, \mathrm{Lid}=30 \%, \operatorname{Span} 80=30 \%)$ & $34.6 \pm 2.6$ & $35.4 \pm 4.0$ \\
\hline
\end{tabular}

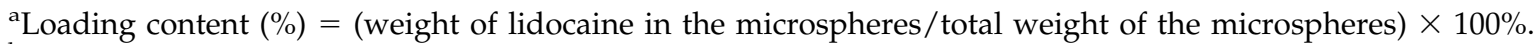

${ }^{\mathrm{b}}$ Encapsulation efficiency was defined as the weight percentage of the loaded lidocaine relative to the initial dissolved lidocaine in the organic phase.

${ }^{\mathrm{c}}$ The emulsion was stirred for $30 \mathrm{~min}$ followed by rotary evaporation of the solvent for $20 \mathrm{~min}$.

${ }^{\mathrm{d}}$ The emulsion was stirred for 30 min followed by rotary evaporation of the solvent for 20 min with gradually heating up to $45^{\circ} \mathrm{C}$. The Lid/PLA ratio was maintained at $150 \%$.

'The emulsion was stirred for $2 \mathrm{~h}$ to naturally evaporate the solvent. The Lid/PLA ratio and Span 80/PLA ratio were maintained respectively at 150 and $15 \%$.
} 


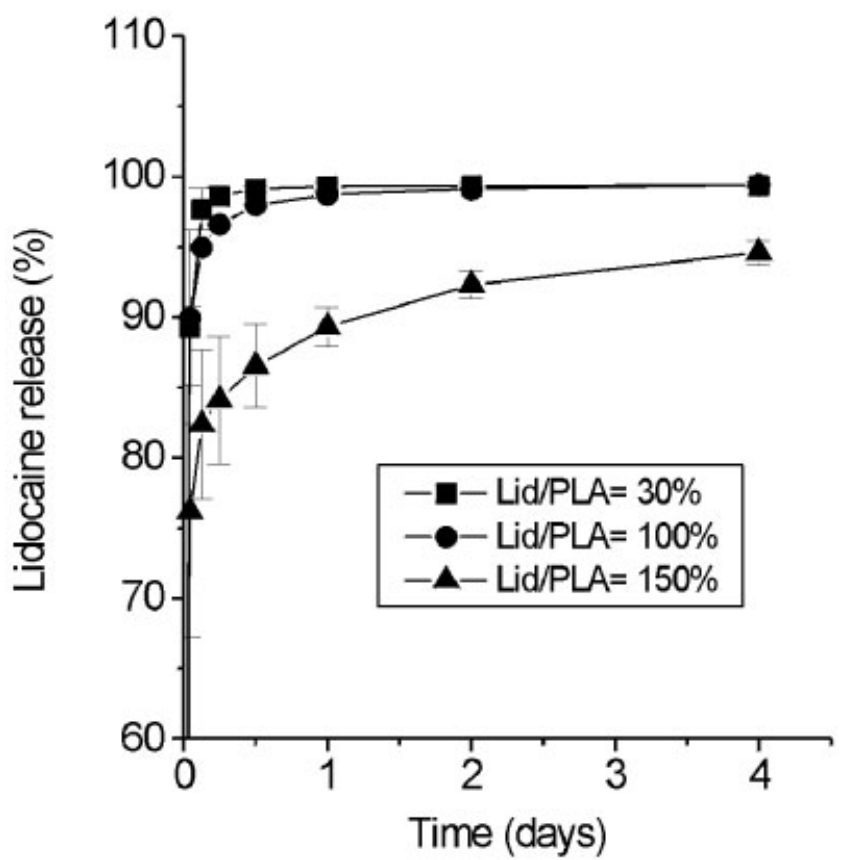

Figure 2. Cumulative release of lidocaine (\%) from microspheres with a Lid/PLA ratio of: $(\boldsymbol{\square}) 30 \% ;(\mathbf{O}) 100 \%$; and $(\boldsymbol{\Delta})$ $150 \%$. Experiments conducted with 30, 100, and 150\% Lid/ PLA ratio corresponded to Experiments 1, 2, and 3 described in Table I, respectively.

\section{Optimization of microsphere formulation}

Effect of lid/PLA ratios

The effects of altering the Lid/PLA ratio (see Experiments 1 to 3 in Table I) of the formulation on lidocaine release are shown in Figure 2. The release rate was significantly reduced when the Lid/PLA ratio was increased from 100 to $150 \%$; primarily due to the crystallization of lidocaine within the microspheres. ${ }^{18}$ This finding was further confirmed in the later DSC studies. In principle, in the presence of crystals, lidocaine release was governed by two sequential steps: (1) dissolution of the crystals, and (2) diffusion of the dissolved lidocaine out of the microspheres. Owing to this requirement of an additional crystal dissolution step, lidocaine release was substantially retarded for microspheres with a high loading content where lidocaine crystals were formed.

Effect of Span80/PLA ratios

To further regulate the rate of lidocaine release, Span 80 was added during formulation of the microspheres (see Experiments 4 to 6 in Table I). Figure 3 shows that a slower release rate was obtained when the amount of Span 80 was increased. This reduction in release rate was believed to be due to the creation of a hydrophobic environment by Span80, which subsequently hindered hydration of the microspheres. As is known, water permeation into the microspheres is essential for the lidocaine diffusion out of the microspheres.

Effect of initial PLA concentrations

To examine the effect of PLA concentration on lidocaine release, the initial concentration of PLA in the organic phase was doubled. To maintain the same Lid/PLA and Span 80/PLA ratios, the concentrations of lidocaine and Span80 were also doubled accordingly (see Experiments 7 and 8 in Table I). As shown in Figure 4, the release rate of lidocaine decreased when increasing the initial PLA concentration. In addition, the degree of burst release of lidocaine (within the first $12 \mathrm{~h}$ ) also decreased from 70 to $40 \%$ when the initial concentration of PLA was raised from 10 to $20 \%$. The reduction in release rate was believed to be due to the formation of a denser microsphere structure when a higher PLA concentration was employed during microsphere formation.

\section{Fusion of the microspheres}

Under a microscope, it was observed that approximately $15 \%$ of the microspheres aggregated or, in-

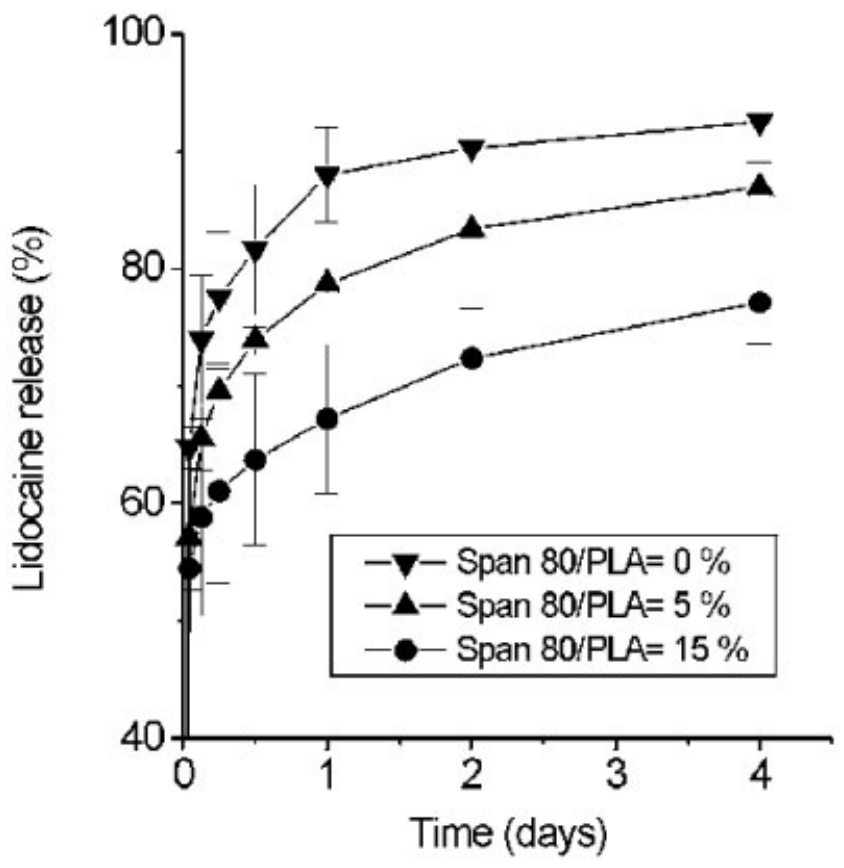

Figure 3. Cumulative release of lidocaine (\%) from microspheres with a Span 80/PLA ratio of: $(\boldsymbol{\nabla}) 0 \% ;(\boldsymbol{\Delta}) 5 \%$; and $(\mathbf{O})$ $15 \%$. Experiments conducted with the 0,5, and 15\% Span $80 /$ PLA ratio corresponded to Experiments 4, 5, and 6 described in Table I, respectively. 


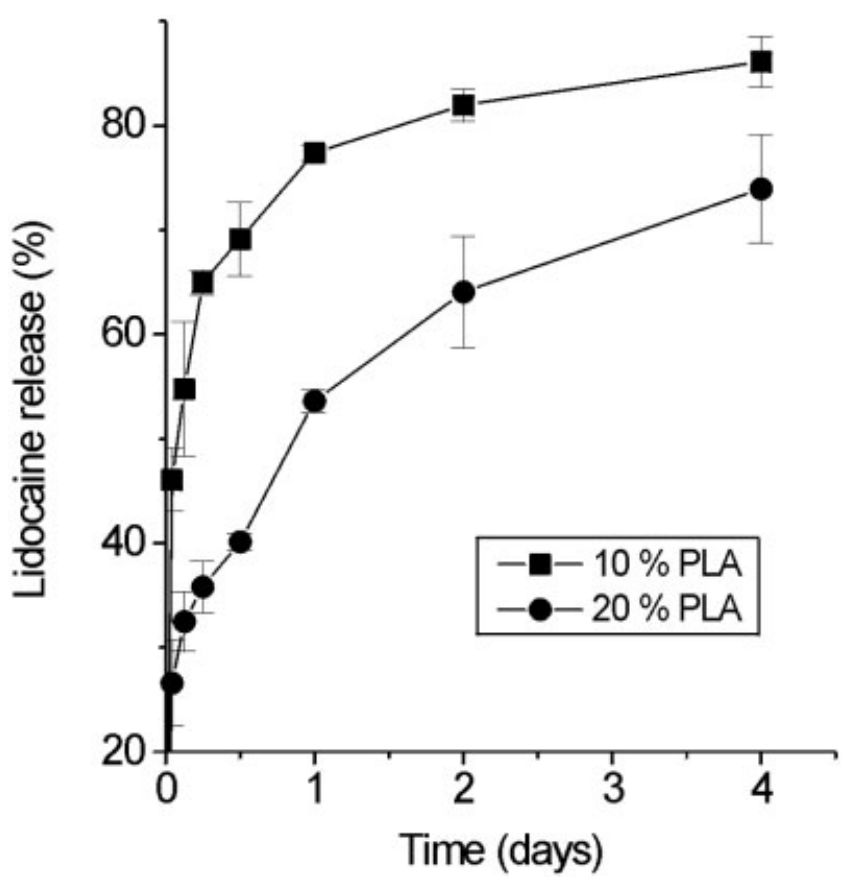

Figure 4. Cumulative release of lidocaine (\%) from microspheres with a PLA concentration (wt/v \%) in the organic

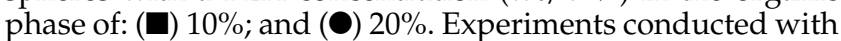
the 10 and 20\% PLA concentration corresponded to Experiments 7 and 8 described in Table I, respectively.

deed, fused to form particles of irregular shapes during the freeze-drying process. The occurrence of this fusion was far less significant if lidocaine was not used or if poly(vinyl alcohol) rather than PO188 was used as an emulsifier during fabrication of the microspheres. Therefore, it was speculated that fusion of the microspheres was caused by either lidocaine or PO188, or by both. Entrapment of a small hydrophobic molecule like lidocaine may have exerted a plasticizing effect on PLA, causing the microspheres to lose their mechanical resistance. The presence of PO188 (PEG-PPG-PEG copolymer) emulsifier in the interphase may also render the microsphere surface to possess a brush-like structure (known to be formed by PEG), which, upon freeze drying, could lead to entanglement of the PEG chains ${ }^{19}$ between different particles, and consequently, aggregation.

During storage of the microspheres, it was also revealed that temperature increase would cause the fusion. To further validate our theory, DSC studies were conducted on microspheres with different lidocaine loadings. Figure 5 shows that the glass transition temperature $\left(T_{g}\right)$ of the microspheres decreased as the lidocaine loading increased, supporting our theory that lidocaine was exerting a plasticizing effect on the PLA-based microspheres. This plasticizing effect would change the microspheres from a glassy to a rubbery state, where the transitional, rotational, and vibrational energies of the polymer molecules were all enhanced. As a consequence, the lidocaine-loaded microspheres would yield a reduced $T_{g}$ accompanied with a loss of their mechanical resistance, rendering fusion of the microspheres to occur. To prevent this event, the microspheres need to be stored at $4^{\circ} \mathrm{C}$ under vacuum immediately following their fabrication. It is also interesting to note that, in agreement with findings by other investigators, ${ }^{18}$ only one peak was observed in the DSC diagrams of microspheres containing no or little (11\%) lidocaine [Fig. 5(c and d), respectively], whereas two peaks were observed in the microspheres containing high $(35 \%)$ lidocaine [Fig. 5(e)]. The former could be explained by the formation of a homogeneous matrix with lidocaine being evenly distributed within the microspheres, whereas the latter was probably due to the occurrence of a heterogenous matrix consisting of the PLA-dispersed lidocaine blends [the first peak in Fig. 5(e)] and the lidocaine crystals [the following peak in Fig. 5(e)].

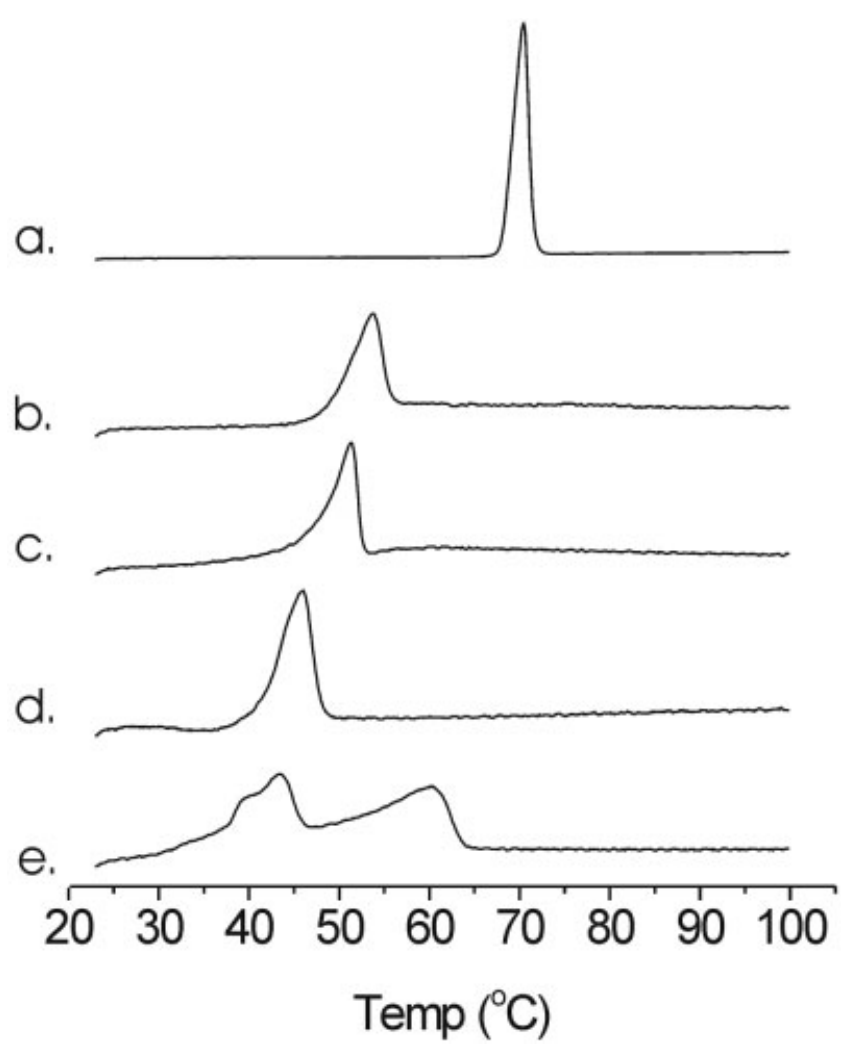

Figure 5. The differential scanning calorimetry diagrams of: (a) lidocaine, $T_{m}=68^{\circ} \mathrm{C}$; (b) PLA, $T_{g}=50^{\circ} \mathrm{C}$; (c) lidocaine-free microspheres, $T_{g}=48^{\circ} \mathrm{C}$; (d) microspheres with a lidocaine loading of $11 \%, T_{g}=41^{\circ} \mathrm{C}$; and (e) microspheres with a lidocaine loading of $35 \%, T_{g}=37^{\circ} \mathrm{C}$ (the first peak from the left). $T_{m}$ and $T_{g}$ were defined as the temperature at the onset of the peaks. 


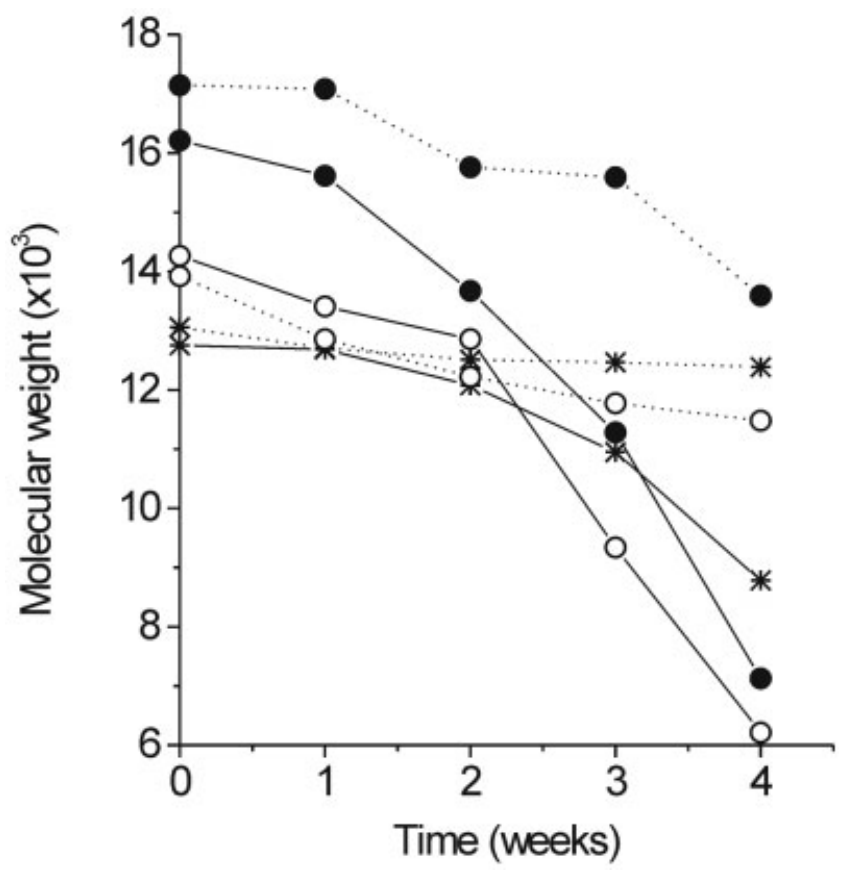

Figure 6. In vitro degradation kinetics of: (-, solid line) lidocaine-loaded microspheres; and (---, dashed line) lidocaine-free microspheres. Molecular weights were determined by gel permeation chromatography. $M_{w}(\mathbf{0}), M_{p}\left(^{*}\right)$, and $M_{n}(\bigcirc)$ represent the weight-averaged molecular weight, molecular weight at the peak, and number-averaged molecular weight, respectively. Microspheres were prepared according to the conditions described in Experiment 8 in Table I.

\section{In vitro degradation of PLA microspheres}

\section{Change in molecular weight}

Figure 6 shows that the lidocaine-loaded microspheres (curves with solid lines) exhibited a faster degradation rate than lidocaine-free microspheres; as their molecular weight was reduced by $50 \%$ within 1 month of incubation compared to only $15 \%$ for the lidocaine-free microspheres (curves with dotted lines). Because ester bonds are susceptible to hydrolysis under nonneutral $\mathrm{pH}$ conditions, degradation of the PLA polymer can be catalyzed by the terminal carboxylic groups produced by the hydrolysis of the ester bonds; a process known as autocatalysis. ${ }^{20}$ In this regard, the acidic or basic nature of the encapsulated drug can also alter the degradation kinetics of PLA. The overall degradation rate, therefore, depends on the $\mathrm{pH}$ balance between PLA and the encapsulated drugs inside the microsphere. In the case of our lidocaine-loaded microspheres, the number of lidocaine molecules was many folds in excess over that of the carboxylic groups, which existed only at the end of the polymer chains. A basic microenvironment produced by the basic lidocaine within the microspheres catalyzed the cleavage of the ester bonds more rapidly than that by the carboxylic groups alone in the lidocaine-free microspheres. As a consequence, the lidocaine-loaded microspheres exhibited a more rapid degradation rate than the lidocaine-free microspheres, as reflected by a greater decrease in the molecular weights of PLA. For comparison, molecular weights of the commercial PLA were also determined, The $M_{p}, M_{w}$, and $M_{n}$ of the commercial PLA were estimated to be $13,138,16,910$, and 14,159 Daltons, respectively. No significant changes in molecular weights between the commercial PLA and microspheres were observed, indicating that no major PLA degradation occurred during preparation of the microspheres.

\section{Change in morphology}

Figure 7 presents the scanning electron micrographs of the microspheres during the degradation process. The lidocaine-free microspheres displayed an intact surface after 2 weeks of incubation [Fig. 7(a)] and a relatively smooth surface after 4 weeks of incubation [Fig. 7(b)]; reflecting a slow degradation rate for these microspheres. In contrast, the lidocaine-loaded microspheres exhibited a smooth surface only after 3 days of incubation [Fig. 7(c)], a rough surface after 2 weeks [Fig. 7(d)], and a beehive shape after 4 weeks of incubation [Fig. 7(e)]; implicitly reflecting a rapid degradation. In addition, more than $95 \%$ of the lidocaineloaded microspheres were fused into irregular-shape particles after 3 days, and only a small fraction of the microspheres remained intact in their structures [see Fig. 7(c)]. Nevertheless, the lidocaine-loaded microspheres appear to be well suited for the proposed use in postoperative pain treatment, because surgical removal of the implanted microspheres could be avoided due to their rapid degradation rate.

In summary, we demonstrated that by a stepwise modification of the formulation of lidocaine-loaded PLA-based microspheres, including optimization of the lidocaine/PLA ratio and initial PLA concentration, as well as incorporation of the Span80, a sustained release of lidocaine over a period of several days could be achieved. In vivo studies on sciatic nerve blocked rats by utilizing the optimized microspheres developed in this study are currently in progress in our laboratories.

The authors would like to thank Professor Richard Laine's group in the Department of Macromolecule at the University of Michigan for their technical support, Professor David Martin in the Department of Material Science and Engineering for his advice. The helpful discussion from Professor Steven Schwendeman's group at the College of Pharmacy and the review of manuscript by Mr. Joseph Yang and Dr. Lai Ming Lee are also acknowledged. 

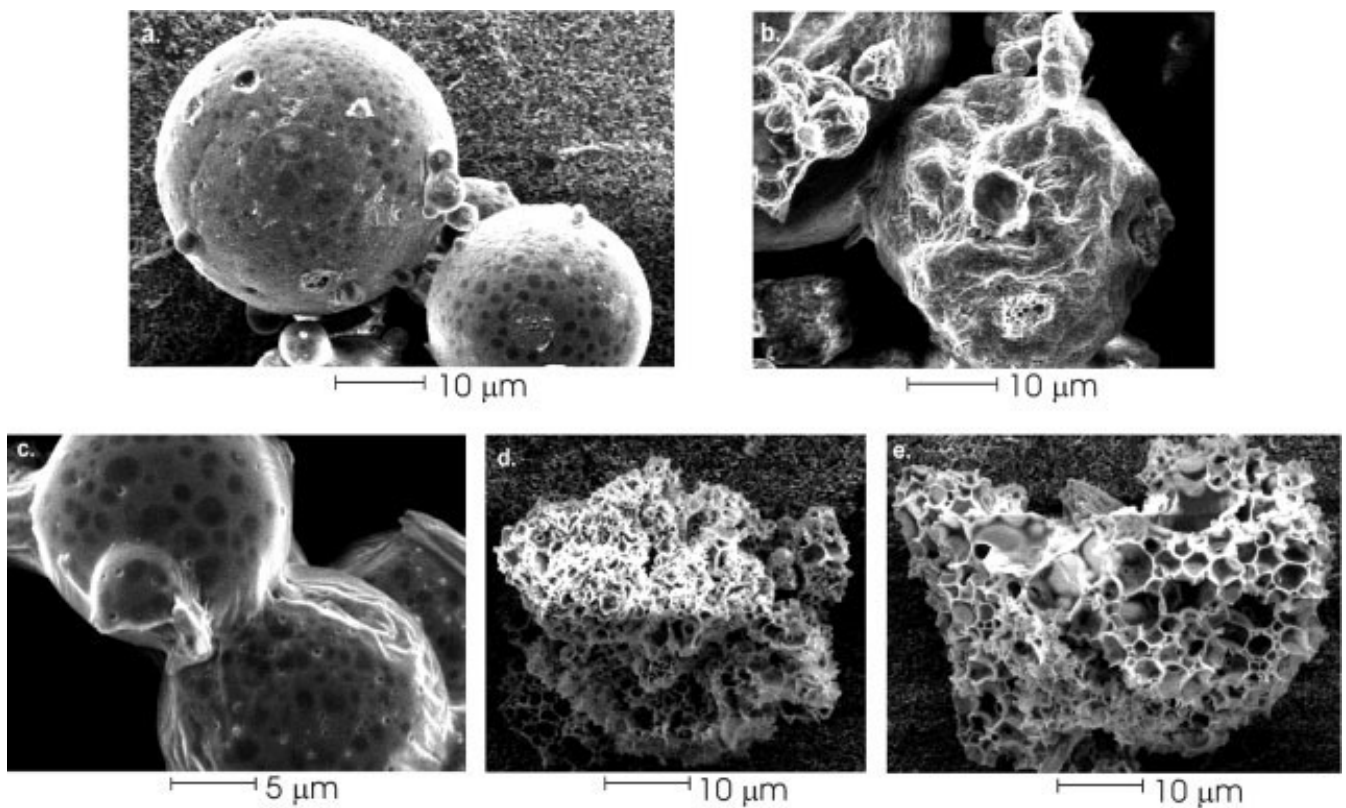

Figure 7. Morphology changes observed by SEM in the in vitro degradation studies of the "lidocaine-free microspheres" at: (a) week 2; and (b) week 4; as well as the "lidocaine-loaded microspheres" (prepared according to the conditions described in Experiment 8 in Table I) at: (c) day 3; (d) week 2; and (e) week 4.

\section{References}

1. Ferrante FM, VadeBoncouer TR. Postoperative pain management. New York: Churchill Livingstone; 1993.

2. Hecker BR, Bjurstrom R, Schoene RB. Effect of intercostal nerve blockade on respiratory mechanics and $\mathrm{CO}_{2}$ chemosensitivity at rest and exercise. Anesthesiology 1989;70:13-18.

3. O'Kelly E, Garry B. Continuous pain relief for multiple fractured ribs. Br J Anaesth 1981;53:989-991.

4. Matthews PJ, Govenden V. Comparison of continuous paravertebral and extradural infusions of bupivacaine for pain relief after thoracotomy. Br J Anaesth 1989;62:204-205.

5. Miller L, Gertel M, Fox GS, MacLean LD. Comparison of effect of narcotic and epidural analgesia on postoperative respiratory function. Am J Surg 1976;131:291-294.

6. Raj PP, Knarr DC, Vigdorth E, Denson DD, Pither CE, Hartrick $\mathrm{CT}$, Hopson CN, Edstrom H. Comparison of continuous epidural infusion of a local anesthetic and administration of systemic narcotics in the management of pain after total knee replacement surgery. Anesth Analg 1987;66:401-406.

7. Cousins MJ, Bromage PR. Epidural neural blockade. In: Cousins MJ, Bridenbaugh PO, editors. Neural blockade in clinical anesthesia and management of pain. Philadelphia: JB Lippincott; 1988. p 253.

8. Raj PP, Denson DD. Prolonged analgesia technique with local anesthetics. In: Raj PP, editor. Practical management of pain. Chicago: Year Book; 1986. p 687.

9. Bromage PR, Pettigrew RT. Crowell DE. Tachyphylaxis in epidural analgesia: I. Augmentation and decay of local anesthesia. J Clin Pharmacol J New Drugs 1969;9:30-38.

10. Renck H, Edstrom H. Thoracic epidural analgesia III. Prolongation in the early postoperative period by intermittent injections of etidocaine with adrenaline. Acta Anaesthesiol Scand 1977;20:104-110
11. Renck H, Edstrom H, Kinnberger B, Brandt G. Thoracic epidural analgesia II. Prolongation in the early postoperative period by continuous injection of $1 \%$ bupivacaine. Acta Anaesthesiol Scand 1976;20:47-56.

12. Wust HJ, Liebau U, Richter O, Strasser K. Tachyphylaxis in continuous epidural anaesthesia with bupivacaine $0.125 \%$ and 0.25\%. Anasth Intensivther Notf Med 1980;15:159.

13. Sjogren S, Wright B. Blood concentration of lidocaine during continuous epidural blockade. Acta Anaesthesiol Scand Suppl 1972;46:51-56.

14. Gorner T, Gref R, Michenot D, Sommer F, Tran MN, Dellancherie E. Lidocaine-loaded biodegradable nanospheres. I. Optimization Of the drug incorporation into the polymer matrix. J Controlled Release 1999;57:259-268.

15. Le Corre P, Rytting JH, Gajan V, Chevanne F, Le Verge R. In vitro controlled release kinetics of local anaesthetics from poly(D,L-lactide) and poly(lactide-co-glycolide) microspheres. J Microencaps 1997;14:243-255.

16. Benita S, editor. Microencapsulation. New York: Marcel Dekker; 1996.

17. Vandewitte P, Esselbrugge H, Peters AMP, Dijkstra PJ, Feijen J, Groenewegen RJJ, Smid J, Olijslanger J, Schakenraad JM, Eenink MJD, Sam AP. Formation of porous membranes for drug delivery systems. J Controlled Release 1993;24:6178.

18. Polakovic M, Gorner T, Gref R, Dellancherie E. Lidocaine loaded biodegradable nanospheres. II. Modeling of drug release. J Controlled Release 1999;60:169-177.

19. Kato T. Aggregation behaviors in aqueous-solutions of amphiphiles studied by light-scattering and pulsed-gradient spinecho methods. Nippon Kagaku Kaishi 1993;7:793-806.

20. Vert M, Mauduit J, Li S. Biodegradation of PLA/GA polymers: Increasing complexity. Biomaterials 1994;15:1209-1213. 